\title{
JPhylolO: a Java library for event-based reading and writing of different phylogenetic file formats through a common interface
}

\author{
Ben C. Stöver ${ }^{*} \mathbb{D}$, Sarah Wiechers and Kai F. Müller
}

\begin{abstract}
Background: Today a variety of phylogenetic file formats exists, some of which are well-established but limited in their data model, while other more recently introduced ones offer advanced features for metadata representation. Although most currently available software only supports the classical formats with a limited metadata model, it would be desirable to have support for the more advanced formats. This is necessary for users to produce richly annotated data that can be efficiently reused and make underlying workflows easily reproducible. A programming library that abstracts over the data and metadata models of the different formats and allows supporting all of them in one step would significantly simplify the development of new and the extension of existing software to address the need for better metadata annotation.
\end{abstract}

Results: We developed the Java library JPhylo/O, which allows event-based reading and writing of the most common alignment and tree/network formats. It allows full access to all features of the nine currently supported formats. By implementing a single JPhylo/O-based reader and writer, application developers can support all of these formats. Due to the event-based architecture, JPhy/o/O can be combined with any application data structure, and is memory efficient for large datasets. JPhylo/O is distributed under LGPL. Detailed documentation and example applications (available on http://bioinfweb.info/JPhylolO/) significantly lower the entry barrier for bioinformaticians who wish to benefit from JPhylo/O's features in their own software.

Conclusion: JPhylo/O enables simplified development of new and extension of existing applications that support various standard formats simultaneously. This has the potential to improve interoperability between phylogenetic software tools and at the same time motivate usage of more recent metadata-rich formats such as NeXML or phyloXML.

Keywords: Phylogenetic metadata, Data reuse, Data annotation, NeXML, PhyloXML, NEXUS, Phylogenetic tree, Multiple sequence alignment

\section{Background}

The amount of available data in organismic and biodiversity-related disciplines, such as phylogenetics, taxonomy or ecology $[1,2]$ as well as related fields of molecular biology, especially genomics or genome evolution has been growing and continues to grow at an accelerated rate. Among other factors, increasingly cheaper high-throughput sequencing technologies [3],

\footnotetext{
* Correspondence: stoever@bioinfweb.info

Institute for Evolution and Biodiversity, WWU Münster, Hüfferstraße 1, 48149 Münster, Germany
}

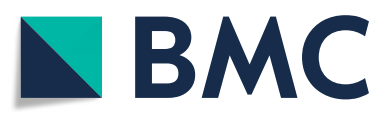

(c) The Author(s). 2019 Open Access This article is distributed under the terms of the Creative Commons Attribution 4.0 International License (http://creativecommons.org/licenses/by/4.0/), which permits unrestricted use, distribution, and

reproduction in any medium, provided you give appropriate credit to the original author(s) and the source, provide a link to the Creative Commons license, and indicate if changes were made. The Creative Commons Public Domain Dedication waiver (http://creativecommons.org/publicdomain/zero/1.0/) applies to the data made available in this article, unless otherwise stated. [4-6], the ongoing digitization of biological collections [7, 8], and large-scale data acquisition (e.g., related to monitoring biodiversity) in citizen-science [9-12] contribute to this increasing amount of primary data. On top of that, the availability of faster processing units allows for increasingly advanced downstream analyses and the parallel application of multiple alternative methods and parameter sets, which in turn leads to even more (derived) data, potentially multiplying the amount with value for reuse in subsequent studies. 
While these developments open up new perspectives for studies and applications that make use of big data, the practical reusability of data continues to be an issue. Primary sequence data tend to be reused on a regular basis, but the accessibility of other derived data types like phylogenetic trees is still low [13]. Phylogenetic hypotheses, for example, are often provided as image files only. Although software exists to reconstruct phylogenetic trees from images [14-17], making trees easily reusable would require representing tree topologies and branch lengths in defined phylogenetic file formats $[13,18]$. Public availability and searchability of scientific data are necessary to foster reuse $[19,20]$ and can be addressed by respective policies of funding agencies and scientific journals [21] together with cyberinfrastructure (e.g., [22-26]) for the long-term storage. Data annotation is an equally important requirement to easily and unambiguously identify and understand relevant data that fits the need for a concrete project $[13,27,28]$. Metadata annotation is necessary to, e.g., unambiguously link tree nodes to sequences in a multiple sequence alignment that was used to generate the tree, or to link tree nodes and sequences to taxonomic information, ideally also using a taxonomic ID (e.g., NCBI Taxonomy [29]) or even better linking a sequence back to the individual specimen it was derived from [30]. Additionally, linking relevant external resources (e.g., voucher information, digitized specimens or sequencing raw data) and providing metadata that reliably identifies the methods that were used to generate data (e.g., the software and parameters used for a phylogenetic inference) would further improve reusability of data and reproducibility of studies. More extensive lists of information to be provided for better reusability and reproducibility can be found in [31, 32]. Storing the results of phylogenetic analyses using metadata-rich formats is an ideal basis to link all necessary metadata and resources.

The big data-driven development of databases to reuse phylogenetic information (e.g., [24-26]) or the application of deep learning approaches (e.g., [33-35]) would be significantly simplified if more data were annotated with more structured metadata and could be automatically collected from a wide variety of studies. Beyond that, making phylogenetic data more reusable is important for every discipline where data is analyzed in a phylogenetic context, evolutionary aspects are part of studies, or alignments by homology or phylogenetic trees are needed at some point during analysis.

Phylogenetic file formats to store taxon or OTU lists, character matrices, multiple sequence alignments or phylogenetic trees and networks, are an integral part of phylogenetic workflows and the basis for interoperability between the software tools and databases involved.
Several formats exist, which can be grouped into two categories: (i) classical formats, such as FASTA, PHYLIP [36], Newick [37] or NEXUS [38] and (ii) more recently developed formats with significantly more advanced metadata models, such as NeXML [39] or phyloXML [40] (See also Table 1). Due to their ability to store rich metadata, the more advanced formats are much better suited to annotate data in a way that allows efficient reuse and clear reproducibility, as argued above. The metadata model of $N e X M L$, for example, uses RDFpredicates from externally defined ontologies to link external resources, allowing maximal reusability of phylogenetic data $(R D F=$ Resource Description Framework, a semantic web technology to formulate logical statements, [41, 42]). These modern formats are $X M L$-based and therefore well-defined by $X M L$ schema definitions. $X M L$ libraries available for nearly all programming languages can be used to process them. In contrast, the classical formats are plain text-based and usually do not have a formal definition (e.g., a grammar), resulting in different dialects and incomplete reader implementations. This frequently leads to interoperability issues when exchanging data between different software, which is another downside of using the classical formats instead of the more recent ones. In practice though, the classical formats dominate and only a small number of researchers actually make use of these newer formats and annotation standards. One important reason for this is that the majority of widely used computer applications only support the classical formats.

To help with the transition of the community to providing richly annotated data, software is required that simplifies such annotation and supports the modern formats. This software should allow importing from and exporting to the classical formats to provide downwards compatibility and be interoperable with applications that do not (yet) support metadata-rich formats. The development of such software can be costly and complicated since different readers and writers for all formats need to be implemented and the representation of the applications' data in the different formats must be developed, which usually requires detailed knowledge on all formats. Since the necessary resources would have to be subtracted from working on the core functionality of the application, this is usually omitted. To foster the development of new and the extension of existing software to support the necessary variety of formats, we created the Java programming library JPhyloIO. It allows access to phylogenetic data through one common interface that fully models the data and metadata concepts of all mentioned formats. This enables Java application developers to support both modern metadata-rich formats (to produce easily reusable data) and classical formats (for larger interoperability) by implementing only one 
Table 1 Formats supported by JPhylolO

\begin{tabular}{|c|c|c|c|c|c|c|c|c|c|c|}
\hline Format & Type & OTUs & MSAs & Trees & Networks & Simple Metadata ${ }^{1}$ & Complex metadata $^{2}$ & Ontologies $^{3}$ & Read & Write \\
\hline FASTA & Text & & $x$ & & & & & & $x$ & $x$ \\
\hline PHYLIP & Text & & $x$ & & & & & & $x$ & $x$ \\
\hline Relaxed PHYLIP & Text & & $x$ & & & & & & $x$ & $x$ \\
\hline NEXUS & Text & $x$ & $x$ & $x$ & & $x$ & & & $x$ & $x$ \\
\hline Newick & Text & & & $x$ & & $x$ & & & $x$ & $x$ \\
\hline eNewick (Newick, NEXUS) & Text & & & $x$ & $x$ & $x$ & & & $x$ & \\
\hline NeXML & $X M L$ & $x$ & $x$ & $x$ & $x$ & $x$ & $x$ & $x$ & $x$ & $x$ \\
\hline phyloXML & $X M L$ & & & $x$ & $x$ & $x$ & $x$ & 3 & $x$ & $x$ \\
\hline MEGA & Text & & $x$ & & & & & & $x$ & \\
\hline$P D E$ & $\mathrm{XML}$ & & $x$ & & & $x$ & & & $x$ & \\
\hline XTG & XML & & & $x$ & & $x$ & & & $x$ & \\
\hline
\end{tabular}

A variety of file formats used in phylogenetics are supported. These can either be based on $X M L$, or define custom types of structured text which is indicated in the second column. The central columns show whether a format supports taxon/OTU lists, multiple sequence alignments, phylogenetic trees or networks and what type of metadata can be attached to at least some of these elements or their subelements. As shown in the two rightmost columns, JPhylo/O can read and write many of the common formats, while formats specific to single applications can only be read

${ }^{1}$ Attaching simple numeric or textual values to data elements

${ }^{2}$ Attaching complex metadata elements that may be represented as $X M L$ structures

${ }^{3}$ Simple annotations can be linked using CURIE-like identifiers and custom $X M L$ tags can be added to all elements but no explicit reference to external ontologies is currently supported in phyloXML

JPhyloIO-based reader and writer. JPhyloIO models data and metadata in all formats (as far as they allow this) without the need for the developer to explicitly deal with this problem, which we hope will help encourage the use of modern metadata-rich formats.

\section{Implementation}

JPhyloIO offers a general way for reading and writing various phylogenetic file formats, without imposing any constraints on the data model of applications using the library. (See Fig. 1 and Fig. 2.) An event-based architecture (similar to iterator-based StAX for XML parsing that is common in Java) was chosen over a model-based approach (that would define its own data model classes), because representing phylogenetic data as a sequence of event objects allows compatibility with all application data structures and memory efficient processing. The JPhyloIO readers and writers for different formats all implement a common interface (using the strategy pattern [43]) and instances of them are created using a factory implementation [43] that can guess the format from a file or input stream.

\section{Event streams for reading documents}

JPhyloIO's reader classes translate the hierarchical data structure of a document with phylogenetic data into a linear sequence of event objects, which is formally described by the grammar in Fig. 3. Events representing data elements that consist of smaller parts (e.g., an alignment that consists of sequences) are modelled as a pair of a start and an end event. The subsequence between such two consists of events that model the content of the data element at hand. By applying this recursively, the hierarchical structure of a document can be serialized to a linear event stream, as shown in the example in Fig. 4. Applications using JPhyloIO need to implement a reader for processing the encountered events and storing relevant information in their data structure (Fig. 1, Fig. 2). This can be done by iterating over the event stream using (StaX-like) pull parsing, which allows the application to actively request events one by one and therefore keep the control flow. Classes for (SAX-like) push parsing are additionally available, if an inversion of control is beneficial, e.g., if multiple event listeners need to be present on the application side. One such application reader implementation allows access to all supported formats, even when additional formats are added in future releases of JPhyloIO, without the need for any format-specific logic.

All created event objects have a string ID, which is unique inside a document's event stream and allows to link events to one another (e.g., a tree node to an OTU). References will only be made to previous events that are already known to the application. To reduce the amount of work for application developers, the minimum information is always directly contained in an event object, so that only more complex application models will need to resolve such ID dependencies.

A web interface that generates lists of JPhyloIO events from any example file is available at http://r.bioinfweb. info/JPhyloIOEventLister. It allows to fold and unfold the output of subsequences and helps to get started with the way JPhyloIO translates phylogenetic data and metadata into events. Besides using custom files, users can also choose from a set of predefined example files in different formats. 


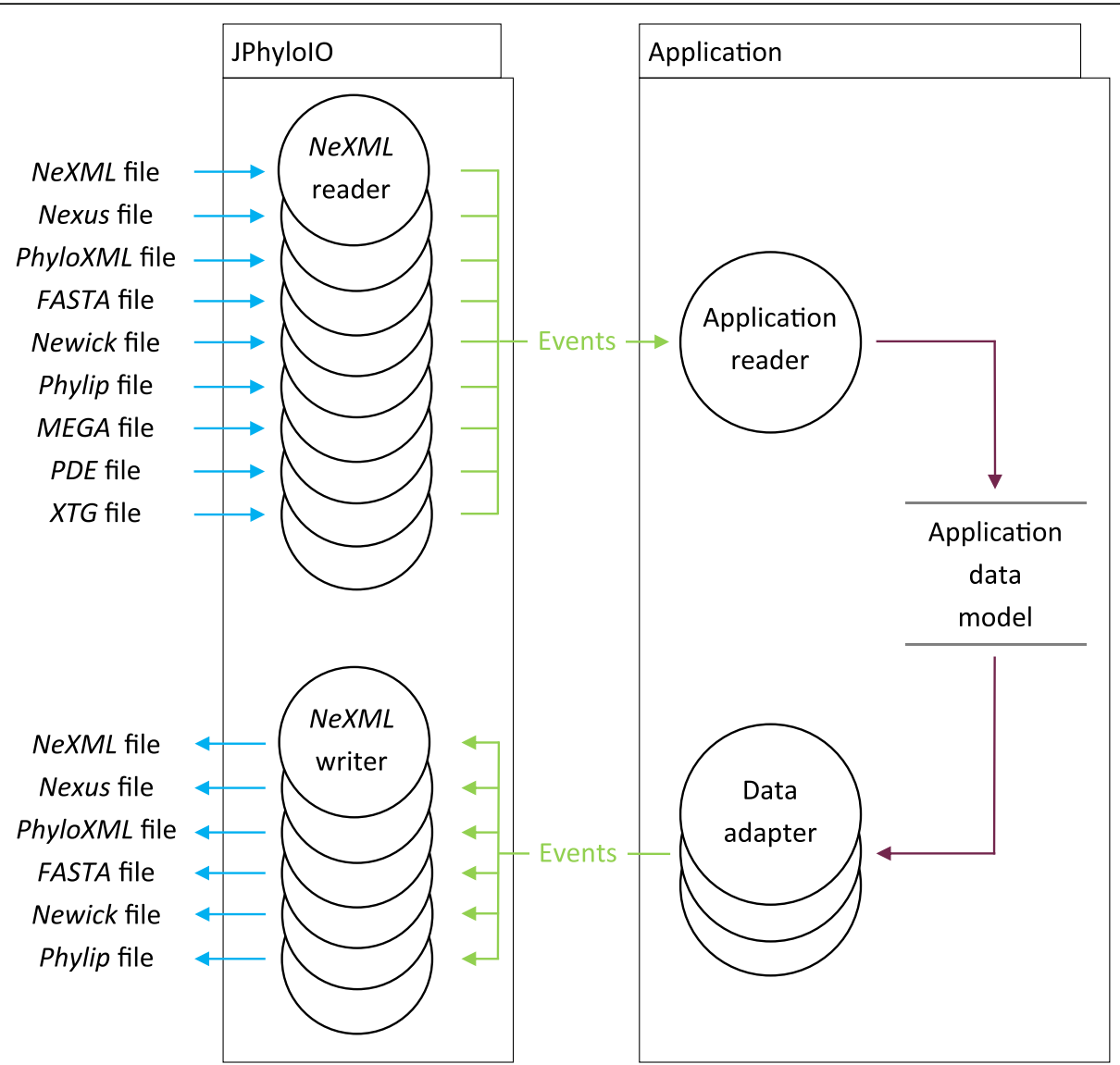

Fig. 1 Data flow diagram showing how data is read into and written from an application data model. JPhylo/O contains a reader for each format that translates the contents of a file to a sequence of events that are then processed by the custom reader of an application. This reader has knowledge of the specific application data model and stores relevant information there. The writers available in JPhylo/O access the contents of that model using data adapters provided by the application that allow random access to the application's data model. (For supported formats specific for a single application, only readers are provided)

\section{Data adapters for writing documents}

Format-independent writing of phylogenetic data cannot be implemented in as straightforward a manner as, e.g., StAX writing for $X M L$, since the required order of the data elements varies between the different target formats, and direct writing of an event stream (as defined by the grammar in Fig. 3) is not possible without having to buffer large amounts of data in some cases. Therefore, we provide adapter interfaces to be implemented by an application. These bridge between the application data model and JPhyloIO writers (Fig. 2, Fig. 5), which then can request certain subsequences of the event stream (which correspond to a grammar node in Fig. 3) in the order required by their target format.

Implementing such data adapters may be slightly more effort for application developers than just writing a method that directly creates an event stream from their data model, but it has the advantage of allowing unbuffered access in the required order for all formats.
Therefore, writing becomes much more memory efficient, especially for large datasets.

\section{Generalization over different metadata concepts}

A key feature of JPhyloIO is that it provides a general way of attaching metadata to any element in a phylogenetic data set, thereby abstracting over different metadata concepts found in the supported formats. In our opinion, the $R D F$-based metadata tags used by NeXML [39] represent the most powerful way of modelling metadata and therefore were chosen as the foundation of our general concept. It allows to link external resources and to represent trees of hierarchical metadata that can be attached to any element of a phylogenetic document. Predicates from externally defined ontologies are used to link data and metadata, which ensures both maximal flexibility regarding the type of metadata and unambiguous and machine-readable descriptions of the relationships at the same time. Technically, RDF distinguishes between "resource metadata elements" that link external 


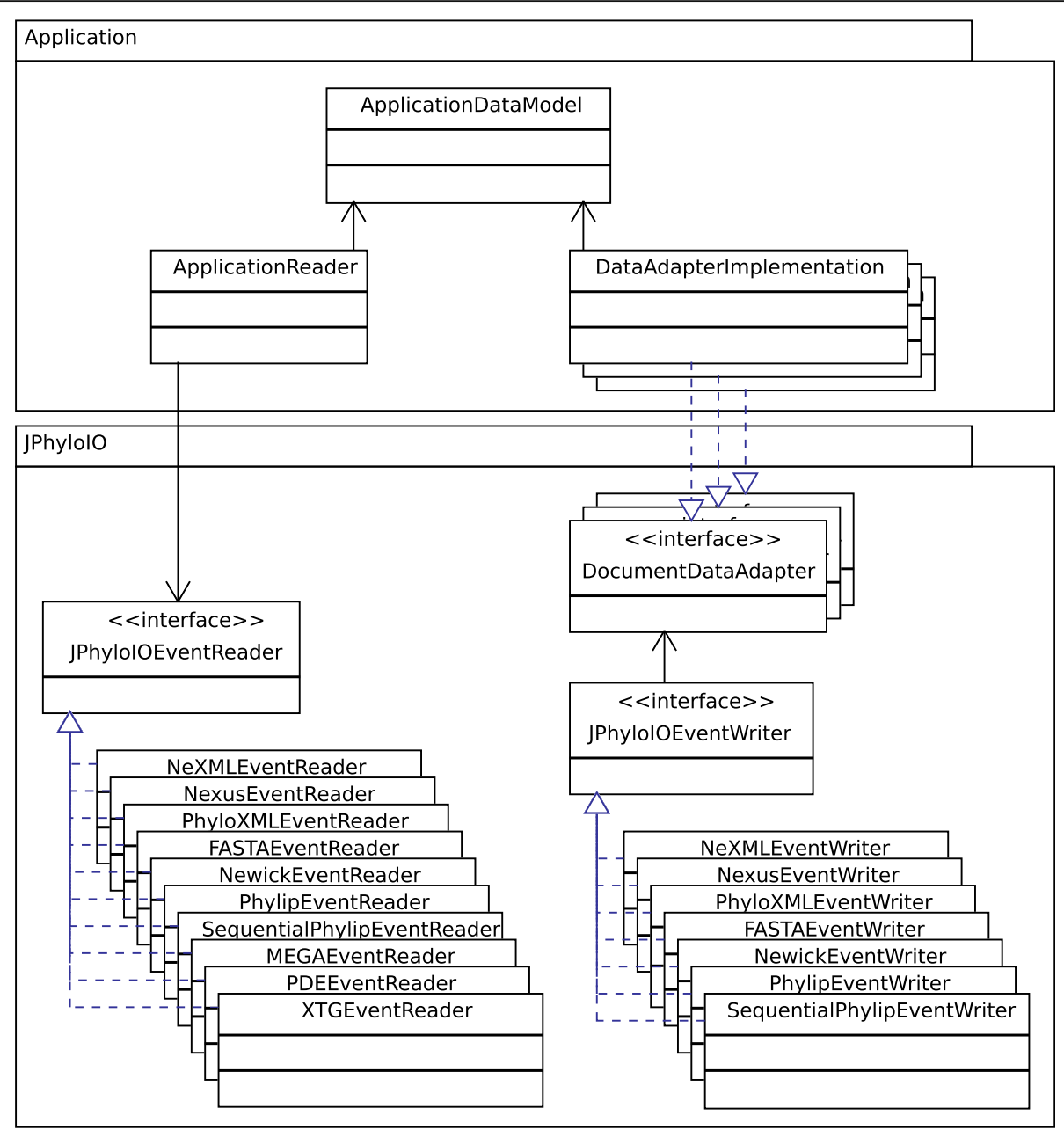

Fig. 2 UML class diagram showing the relation between JPhy/O/O and an application based on it. All readers and writers implement a common interface to be easily exchangeable in the application. Event readers produce a sequence of events (see Fig. 1) processed by an application reader class that acts as an adapter between JPhylo/O and the application data model. Conversely, a set of data adapter implementations of the application allows the JPhylolO writers to access the data. Writing needs a slightly more complex architecture than reading, because writers need to access that data in different orders depending on the target format. To achieve this, a set of data adapters (see Fig. 5 For details) is necessary, each providing a subsequence of the whole event stream modelling a document

resources or a set of other internal metadata elements (forming a subtree within a metadata tree) and "literal metadata elements" that link concrete values (e.g., strings or integers).

Following this structure, JPhyloIO provides a resourceand a literal metadata event class. As shown in Fig. 3, the event grammar allows nesting sequences of metadata events (represented by the grammar node MetaInformation) in all data elements. Metadata can either be represented as a resource- or a literal metadata event. The literal metadata objects may be simple values (e.g., numbers or strings) or complex $X M L$ data, modelled by a sequence of respective events. As an alternative to processing the JPhyloIO event stream directly, our library provides adapter classes between JPhyloIO readers and both iterator- and cursor-based StAX readers and writers to empower application developers to directly reuse possibly existing code for StAX-based reading and writing of respective data without the need for any adaptation to JPhyloIO.

\section{Ways to extend JPhylolO}

All readers and writers in JPhyloIO implement common interfaces and several abstract implementations of these are available that provide shared functionality, e.g., specific for processing text or $X M L$ formats. It is therefore simplified for developers to add new readers and writers for additional (custom) formats that integrate seamlessly with the architecture of the library and can directly be used with all JPhyloIO-dependent code in other software. 


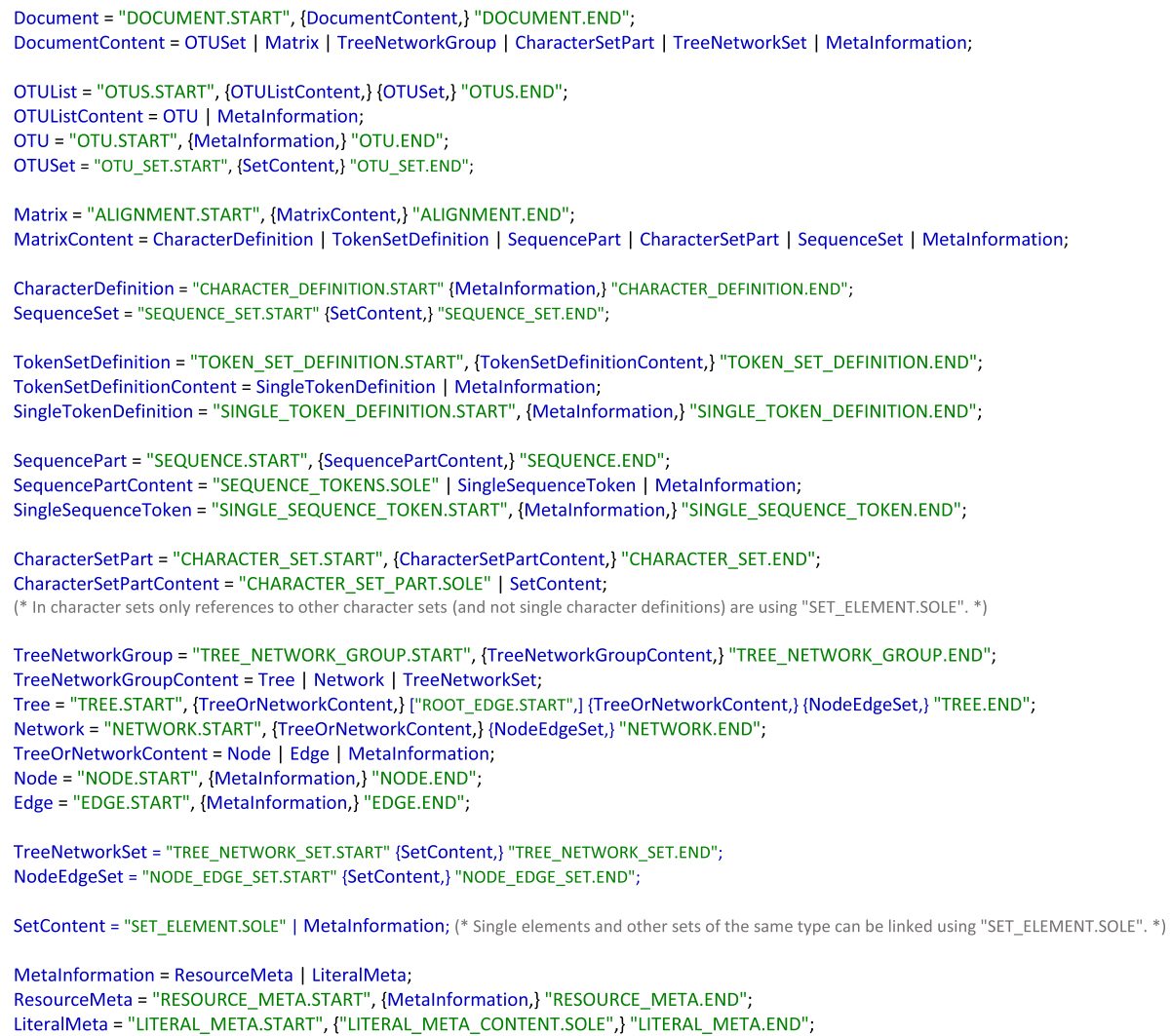

Fig. 3 Grammar describing the event sequence generated by JPhylo/O readers. These readers translate the hierarchical data structure of a phylogenetic file (e.g., a NeXML file consisting of an alignment and a tree, which again consist of sequences or nodes and edges, and so forth) into a sequence of events as defined by this grammar in extended Backus-Naur form (EBNF). The terminal symbols (in green) represent the types of events, each of which either has a single SOLE or a START and END version, depending on whether additional data can be nested or not

For creating complex Java objects from metadata event sequences or to write them back, an interface with a set of default implementations for common types is provided, which can be used for additional custom implementations.

NEXUS-related classes are designed to use individual handlers for all NEXUS blocks and commands, allowing to easily add support for new or custom NEXUS elements in third party modules.

\section{Results}

JPhyloIO is an open-source programming library that allows to read and write different phylogenetic file formats using a single event-based interface as described above. It covers taxon- or OTU lists, character matrices or multiple sequence alignments, phylogenetic trees or networks and sets of elements (e.g., character sets). Simple annotations and more complex metadata can be attached to all elements of a document (e.g., trees, tree branches, sequences) and JPhyloIO translates these using the available features of each supported format.

Source codes and binary distributions are available under the terms of the GNU Lesser General Public
License 3 from http://bioinfweb.info/JPhyloIO/. This website also provides an extensive documentation, including a detailed JavaDoc and a set of example applications.

\section{Supported formats}

As shown in Table 1, JPhyloIO supports reading and writing the majority of phylogenetic file formats, including common extensions of these. Additionally, reading of some application-specific alignment and tree formats is possible. The library imposes no restrictions on alphabets used in molecular, morphological and other character matrices, but guarantees that no invalid output for any of the target formats can be written.

Sequence data, including optional comments, can be read from and written to the FASTA format, with optional column indices at the beginning of each line being processed correctly. Writing of sequences and optional comments is supported, but generated files will never contain column indices, since these are not widely supported and may cause problems in other software.

The PHYLIP format exists in a standard [36] and a relaxed [44] variant, which can both be read in interleaved and non-interleaved forms (the non-interleaved form is 


\section{Document:}

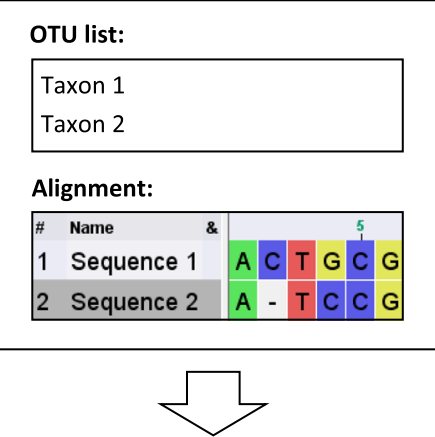

Generated events:

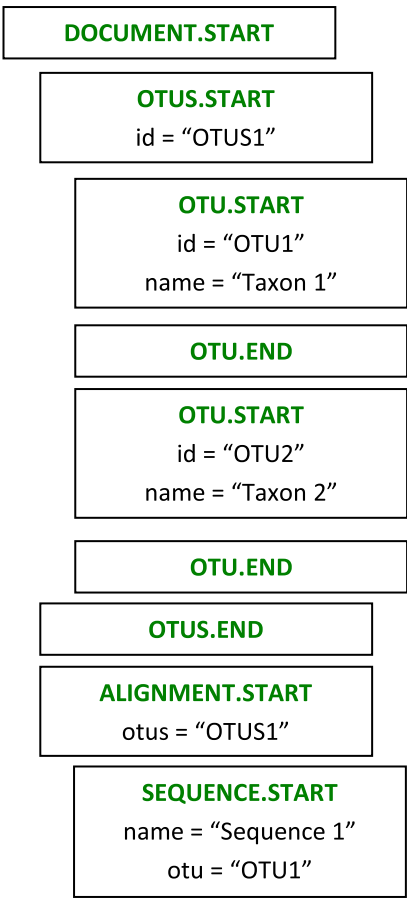

SEQUENCE_TOKENS.SOLE $\{$ "A", “C", “T”, "G", “C", “G” $\}$

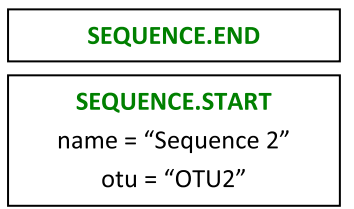

SEQUENCE_TOKENS.SOLE $\{$ "A", “-", "T", “C", “C", “G”

SEQUENCE.END ALIGNMENT.END DOCUMENT.END
Fig. 4 Example document with its respective event sequence. The document contains an OTU list and an alignment, which references this list. The event sequence is generated by a JPhylo/O reader (see also Fig. 2), where each box represents one event. Each has an ID in order to be referenced by subsequent events, as exemplarily shown by the OTU list and OTU start events, which are referenced by the related alignment and sequence start events

written for both). The PHYLIP format allows sequence names only up to a certain length (which can be longer in the relaxed variant), resulting in the need to shorten them by JPhyloIO writers. In contrast to many other available software tools, this implementation ensures that all written names are unique, even if the full names only differ in characters behind the cut-off position. If sequence names were edited, the application will be informed by a translation object, mapping old to new names.

The NEXUS format [38] is a text format consisting of blocks that contain different types of data. Each block consists of a set of NEXUS commands. JPhyloIO offers readers and writers that support commands of the TAXA block containing taxon lists, the DATA, CHARACTERS and UNALIGNED blocks containing sequence and alignment data, the TREES block containing phylogenetic trees and the SETS block, containing sets of other items. One type of custom NETWORKS blocks containing phylogenetic networks in eNewick format is also supported (see below). Sequence data can be in standard or interleaved format with both single character and longer tokens and ambiguous character definitions being supported. Tree nodes can be referenced by the taxon label, the taxon index, or by using a separate translation table. In contrast to other software, JPhyloIO supports all three methods both for internal and terminal tree nodes, while translation can be switched off for internal nodes if necessary, e.g., to avoid conflicts between support values and taxon list indices. For the SETS block, character, taxon and tree sets are currently supported. The DISTANCES, ASSUMPTIONS and NOTES blocks are currently not supported. As NEXUS files identify all elements by a unique label (instead of distinguishing between labels and IDs as, e.g., in $N e X M L$ ), the respective JPhyloIO writer edits labels to be unique if necessary, and reports such changes using the same translation object as the PHYLIP writer described above.

In addition to the initial NEXUS standard, the TITLE and LINK commands from Mesquite [45] that allow linking between blocks (e.g., TAXA blocks can be referenced by CHARACTERS or TREES blocks) and the MIXED sequence datatype extension [46] from MrBayes [47] are recognized.

Phylogenetic trees are represented as Newick strings [37] in the TREES block of a NEXUS document or in 


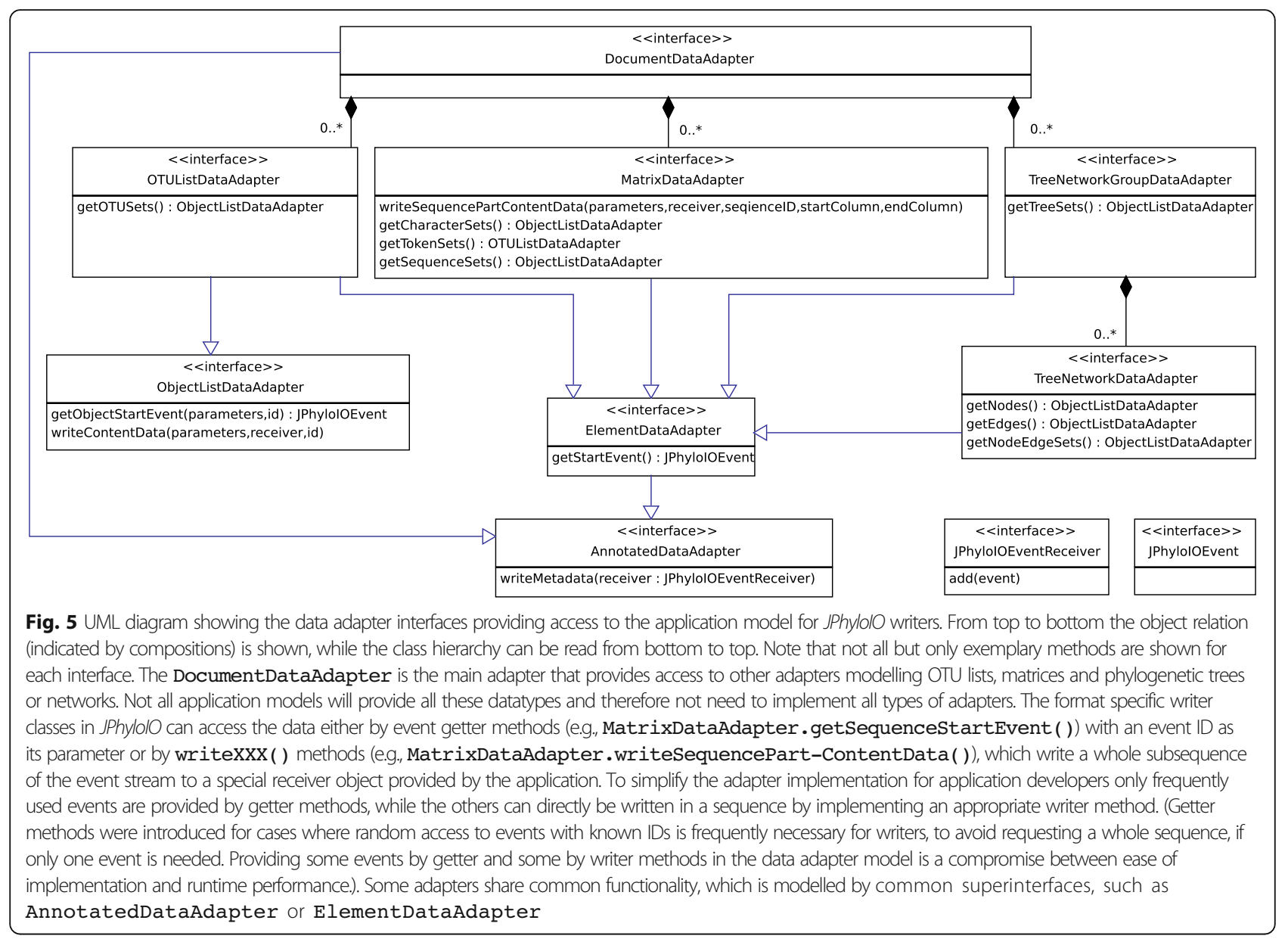

separate text files containing a set of Newick strings separated by semicolons, which are sometimes referred to as Newick files and are, e.g., used by MEGA [48]. Such Newick files are modelled as a separate format in JPhyloIO that can be read and written. Newick tree definitions (in Newick and NEXUS files) may contain metadata in hot comments, which can also be read and written. (See below.)

The readers for both NEXUS and Newick can also read definitions of phylogenetic networks in the Extended Newick or eNewick format [49] and model its crosslink type (if specified) as metadata.

NeXML [39] is a more recent $X M L$ format that is inspired by NEXUS but allows a more advanced way of linking different phylogenetic data elements (e.g., a tree node to an OTU). Additionally, it uses RDFa to attach metadata to all elements (trees, alignments, nodes, sequences, ...), which provides the basis for the general metadata model used in JPhyloIO. (See below.) Readers and writers supporting all features of the format, including its full metadata concept and automated handling of custom sequence tokens, are provided by our library.
phyloXML [40] also models complex metadata using a different concept than $N e X M L$. It is used to store phylogenetic trees and is fully supported by JPhyloIO. Although phyloXML uses a hierarchical tree representation, it allows to specify additional clade relation tags to define phylogenetic networks that are used by JPhyloIO's reader and writer.

In addition, readers for some application specific formats are available. For the MEGA format [48], a reader provides access to its alignment data and character sets (attached by the LABEL, GENE or DOMAIN commands of the MEGA format). Multiple sequence alignments and attached metadata from $P D E$ files produced by the alignment editor PhyDE [50] and trees, including their metadata, from XTG files used by the phylogenetic tree editor TreeGraph 2 [51] can be read as well.

\section{Supported metadata models}

Whereas the metadata representation in NeXML is by definition identical to JPhyloIO's RDF-based metadata model (as described in "Implementation" above), reading and writing of other formats requires a translation to the respective format-specific model. FASTA and PHYLIP do 
not support metadata, so the respective writers ignore provided attachments and log warnings.

phyloXML does not use an RDF-like concept, but offers a fixed set of metadata elements, stored in special $X M L$ tags. To access such metadata in JPhyloIO, we defined $R D F$ predicates for each predefined metadata element for internal use in JPhyloIO, to allow identifying the phyloXML tags in our RDF-based model. In addition, phyloXML offers ways to freely attach metadata by (i) property tags to attach simple annotations (e.g., strings, numeric values or URIs) to trees, clades or sequences and (ii) custom $X M L$ structures added to a whole document, a tree, a clade or some of the predefined annotation tags. JPhyloIO makes use of all these features to attach metadata not linked using phyloXMLspecific predicates. In combination, this allows to read and write all modelled metadata. Since representing custom hierarchical $R D F$ metadata (different from the predefined phyloXML annotation types) is not possible in this format, parts of it will be ignored during writing and respective warnings (similar to FASTA and PHYLIP) will be logged. Different strategies on how to translate a full $R D F a$ annotation tree into phyloXML are offered by JPhyloIO and can be selected using a writer parameter.

For attaching metadata to nodes and branches in Newick strings [37], two extensions that make use of hot comments (comments that contain actual metadata) are supported by JPhyloIO. One is "New Hampshire eXtended" or NHX $[52,53]$, a precursor of phyloXML that allows to use a limited set of its predefined annotations, identified by the respective phyloXML predicates in JPhyloIO.

The other extension, used by, e.g., TreeAnnotator from the BEAST package [54] and recent versions of MrBayes [47], allows to attach numeric or string values (or arrays of these) to nodes and branches using a free string identifier. These identifiers differ from the $R D F$ predicates (used in $N e X M L$ ), since they can have any form and do not need to be URIs. To solve this, all meta-events in JPhyloIO can carry a string identifier and an RDF predicate as alternative descriptions of their relation to their subject. If a string representation is needed for writing and was not provided, the local part of the predicate CURIE will be used.

By supporting these two annotation concepts, JPhyloIO allows to read and write metadata from and to Newick and NEXUS files. As in phyloXML, hierarchical metadata cannot be written and warnings will be logged.

JPhyloIO also reads metadata from the applicationspecific XTG and PDE formats. Both formats may contain a fixed set of metadata for some of their elements and according predicates in namespaces for internal use are defined to identify these (the same way as for phyloXML). The XTG format and TreeGraph 2 [51] additionally provide the functionality to attach numeric or string annotations to each node or branch of a tree using any string identifier, which are also supported. Basic annotations present in the MEGA format (e.g., a description text for a matrix) are read as well.

\section{Code example}

Figure 6 provides example code for writing simple and nested metadata attached to one node and one branch of a tree and shows the output in three different formats that result from it. In addition, the documentation on the JPhyloIO website contains further code examples that are documented in detail and can be downloaded to test and run them. These are available at http://r.bioinfweb.info/JPIODemo.

\section{Ways to get involved}

Feedback and contributions of the community to the project are made possible by the public bug tracking system on the JPhyloIO website, which is also open for feature requests. In addition users can ask questions in the JPhyloIO ResearchGate project, using the support e-mail address on the website or via the bioinfweb Twitter account. Code contributions are possible within the JPhyloIO GitHub repository. (URLs can be found below and on the JPhyloIO website.)

\section{Discussion}

Classic formats like FASTA, PHYLIP or NEXUS still play an important role when working with sequences, alignments and phylogenetic trees, mainly because widely used applications often solely rely on these formats (until now). More modern formats with advanced metadata models like NeXML or phyloXML allow to unambiguously describe and link data and therefore increase its reusability and the reproducibility of underlying studies $[13,32,55]$ in a way that would never be possible using only classical format to represent data. JPhyloIO generalizes over these different types of file formats while still supporting their individual feature sets. Therefore, it allows bioinformaticians to efficiently develop applications that address both the need for interoperability with widely used software by supporting classical formats and the need for better metadata annotation using modern formats at the same time. This can be achieved by just implementing one interface without having to invest additional resources into each supported format. Developers do not even require detailed knowledge about all formats their applications support, since JPhyloIO takes care of which format features to use to optimally represent the content to be written.

Extending existing applications with support for additional, in particular for modern metadata-rich formats, is also simplified by JPhyloIO. It integrates well into 

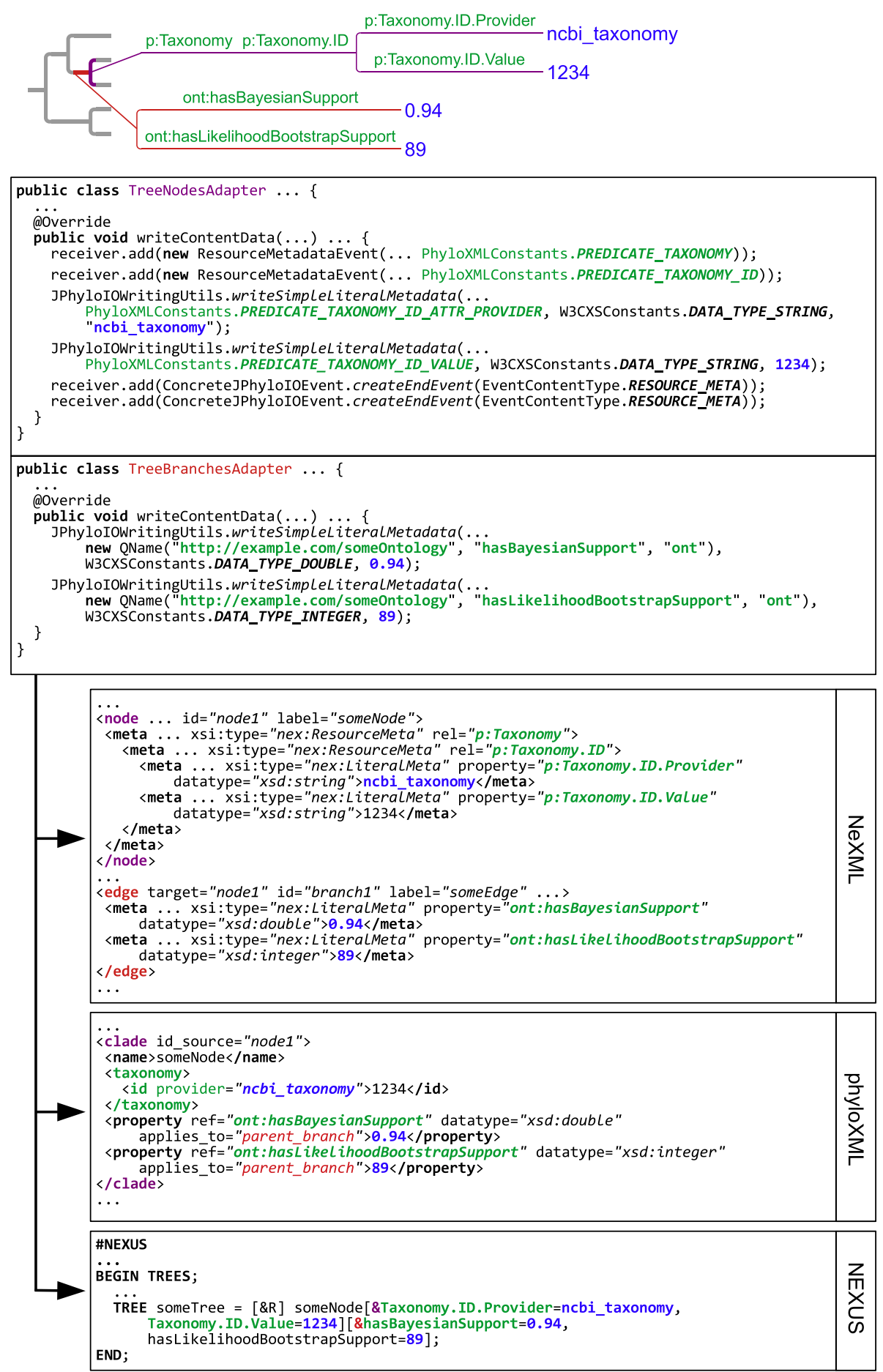

Fig. 6 (See legend on next page.) 


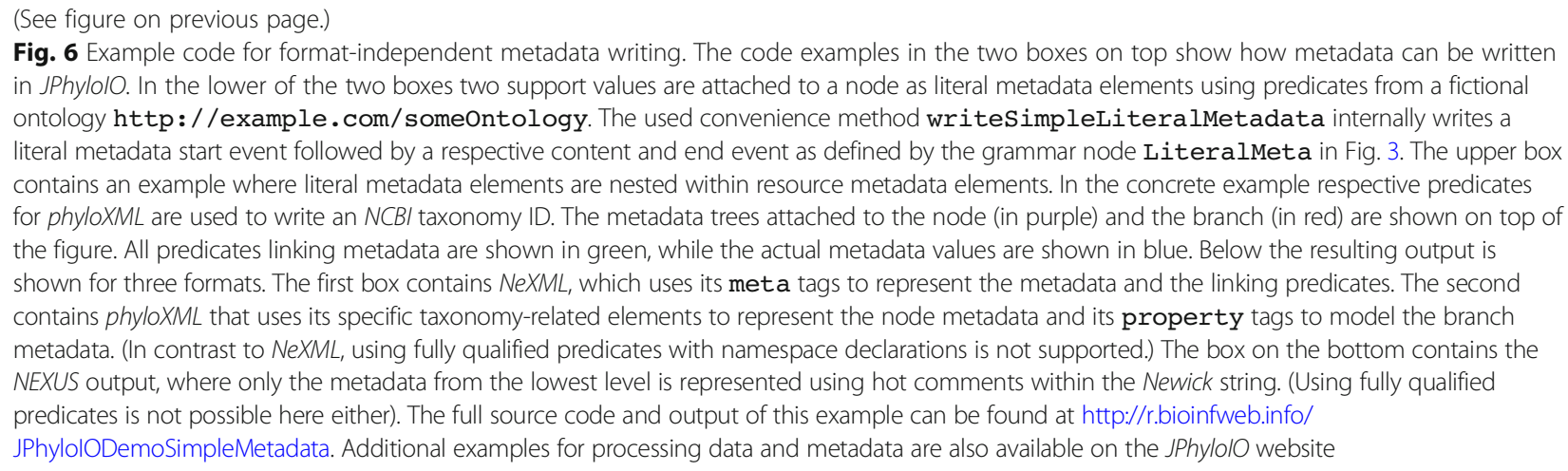

any existing application data model due to its eventbased architecture, which does not impose any requirements on the way data is modelled and stored by an application.

\section{Comparison with other libraries}

Other libraries exist for the Java programming language that support reading or writing of alignment or tree formats. Forester [56] allows to read and write alignments in FASTA, PHYLIP and NEXUS and phylogenetic trees in phyloXML, NEXUS, and NHX. Phylogenies in the Tree Of Life Response Format [57] can be read. The $N e X M L$ format with its powerful metadata model is not supported and no generalization over the different metadata models exists. The tree readers implement a common interface, but there is no such interface for reading or writing trees and alignments together. As a consequence, NEXUS files containing sequence and tree data need to be processed multiple times independently. Unlike JPhyloIO, Forester enforces its own predefined data model, which can have disadvantages for certain use cases as discussed below. The NEXUS TAXA block is only supported when writing trees but not considered for reading trees or for reading and writing alignments, while NEXUS sets are not modelled at all.

In its current version 4.2.7, BioJava [58] includes only readers and writers for sequence data from the FASTA and the GenBank format. The BioJava legacy version 1.9.2 [59] provides an event/call-back based API through a common interface for some sequence formats, among them the alignment formats FASTA and MSF, but none of the other formats supported by JPhyloIO. Independent readers and writers for PHYLIP and NEXUS (including support for trees but not for sets) are available, which cannot be accessed through the event-based API. There is no support for NeXML, phyloXML or complex metadata.

NeXML can of course also be read and written using its reference Java API [60] implemented together with the publication of the format [39] but this library is not intended to support other formats and abstract over their features.

In other languages, multiple format-specific APIs are available (e.g., [61, 62] and many unpublished ones), some of which also generalize over different formats (e.g., [63-65]).

BIO::Phylo [66] is a Perl library that supports a number of alignment and tree formats, among them 6 of the 9 formats supported by JPhyloIO. Reading and writing is possible through a common interface but a predefined data structure is enforced. Metadata connected using $R D F$ predicates is modelled. phyloXML-specific predicates are used in a similar way as in JPhyloIO, while the set of supported elements is less complete (property and clade_relation tags are not, and legal custom tags are only partly supported). BIO::Phylo is able to read (but not write) some types of hot comment tree annotations from NEXUS, while JPhyloIO supports to read and write a larger set of these.

NCL for C++ [64] supports FASTA, Newick, NEXUS and PHYLIP. Plans to support NeXML and phyloXML were announced in 2010, but have not been implemented as of this writing, and therefore complex metadata is not modeled. Hooks for the application to directly process a whole alignment or a whole tree are provided, but these data elements are much larger than in JPhyloIO (where event objects only model, e.g., a short sequence part or a single tree node) and processing of large alignments or trees can be less efficient in NCL.

By making use of the Java Native Interface (JNI) it is possible in principle to access the functionality provided by JPhyloIO from nearly all other programming languages. For some languages, special packages are available to make this more convenient, e.g., Py4J [67] for Python or rJava [68] for $R$. It should be noted that making API calls this way is usually more intricate than working with JPhyloIO in Java directly.

Compared to the existing Java libraries and even libraries in other languages, JPhyloIO supports a large 
number of formats with a more complete coverage of their feature sets. It does that through a single common interface, while allowing memory efficient event-based processing independent of the application's data structure. JPhyloIO's generalization over different metadata models, which allows full access to such data from all formats, is currently not offered by any other Java library. (As mentioned above, BIO::Phylo allows access to a comparable range of formats in Perl but is not event-based.)

\section{Event-based processing versus predefined library data structures}

With an event-based architecture as implemented in JPhyloIO, application developers can decide for each event how long it should be kept in memory or not. Libraries with predefined data structures load all data from a file into memory at the same time, regardless of the application requirements. This is especially inefficient for use cases that do not need random access to all data (e.g., determining the GC-content of large sequence data sets, searching for certain repeat motives in them or counting the occurrences of a certain node in a large set of trees, e.g., taken as samples from Bayesian phylogenetic inference). Event-based processing reduces the amount of memory needed in such cases from $\mathrm{O}(\mathrm{n})$ (linear to the dataset size, e.g., the number of nucleotides) to $\mathrm{O}(1)$ (constant, independent of the dataset size), since only the current or a few recent events need to be in memory at once to perform such tasks.

For applications that need random access (e.g., alignment or tree editors), the event-based architecture is still beneficial, because these are often not interested in the whole content of a file (e.g., only in sequence data but not trees) and therefore can directly discard unused events, which they could not do when using libraryspecific data structures. Even more relevant for complex applications may be the flexibility regarding the data structure. Providing concrete data storage classes with a library, forces applications that need a more advanced or specific model to load the data into instances of library classes first and then copy it into their own specific data structure. This way, the data of at least one file will be in memory twice, which may become a problem for large data sets. Such a problem does not occur with JPhyloIO, since event data can directly be stored into any application-specific data structure.

With this in mind, we acknowledge that predefined model implementations may be beneficial for simpler scripts and tools, because developers will not have to deal with implementing their own data structure. Furthermore, creating an application reader for an event stream will usually require a little more effort than simply fetching information from a predefined library data structure, since the reader will need to keep track of the current state, e.g., the current alignment and sequence, in order to process a sequence tokens event. However, this additional effort will not be significant for the majority of more complex applications that benefit from the memory efficiency and flexibility of the data structure and are the main target for JPhyloIO. It is also easy to combine JPhyloIO with established model standards like the sequence model of BioJava, while it still allows to access data not modelled by such third-party libraries.

\section{Current usage}

JPhyloIO was developed closely together with LibrAlign [69], a Java library providing powerful and reusable GUI components for displaying and editing multiple sequence alignments and attached raw- and metadata. To back its GUI components, LibrAlign provides a fully implemented data model for sequence and alignment data including ready-to-use reader and writer implementations for JPhyloIO.

The Taxonomic Editor of the EDIT platform for Cybertaxonomy [70] manages taxonomic workflows and their data, while persistently linking character data to preserved individual specimens [30]. AlignmentComparator [71] compares alternative multiple sequence alignments of the same dataset. Both make use of JPhyloIO and LibrAlign for reading and writing alignments and attached metadata. LibrAlign and JPhyloIO also provide the basis for the alignment editor PhyDE 2 [72] and they are currently used by our group in the development of tools for the evaluation of automated multiple sequence alignments for phylogenetic purposes and the analysis of microstructural mutational patterns in non-coding DNA.

The tree-related functionality of JPhyloIO is the basis in the ongoing metadata model extension in the phylogenetic tree editor TreeGraph 2 [51]. Versions 2.11.0 and later already use JPhyloIO for importing phylogenetic trees and their metadata from $N e X M L$. Future versions will adopt the $R D F$-based metadata model into the core data model of the application and simplify meaningful annotation of phylogenetic trees and their nodes and branches using metadata linked with predicates from externally defined ontologies. The generalized metadata model of JPhyloIO simplifies importing and exporting metadata for future versions of TreeGraph 2 significantly.

\section{Future development}

JPhyloIO will remain under active development in the future and community contributions are easily possible using GitHub and other platforms, as mentioned above. According to the needs of depending software, the library will be adjusted to future changes of the supported formats and be extended to support additional formats. 
API stability is a key aspect and releases follow the established standard of semantic versioning [73].

As mentioned, the NOTES block of the NEXUS format is currently not supported by JPhyloIO, although it is used by some applications to store metadata related to data from other NEXUS blocks. While the future of metadata representation probably lies in the usage of more advanced formats like NeXML or phyloXML, having downwards compatibility to the NOTES block might still be beneficial. In contrast the metadata models of the other supported formats (including the hot comments in NEXUS), the NOTES block represents a set of metadata elements that usually trail the actual data as a whole and can reference a variety of the previous data elements. In order to model this in JPhyloIO, the event grammar would have to be extended to allow metadata events related to a data event at any later position in the stream instead of requiring it to be nested within the respective data events. This would have made developing application readers for the event stream more complicated. The only option to avoid this is to buffer all data until the NOTES block is read, which would destroy the memory efficiency of event-based processing. Based on the requirements of the applications currently based on JPhyloIO, we preferred a more concise event grammar and memory efficiency over supporting the NEXUS NOTES block. Should the future usage of JPhyloIO impose different requirements we would consider to change that strategy and possibly extend the event grammar, ideally in a downwards compatible way.

In addition to the current abstraction over different formats, the abstraction over (future) metadata ontologies relevant for phylogenetics (e.g., possible in $N e X M L$ ) can become a focus. If a critical number of established ontologies will be present, it may be interesting to extend JPhyloIO to model equivalent or similar predicates in different ontologies to allow translating between them and to access knowledge in a general way.

\section{Conclusion}

The field of phylogenetics as well as biological sciences as a whole would strongly benefit from a more widespread use of data annotation and respective formats. Unambiguously describing and processing morphological characters and states, documenting voucher information in collections, linking raw data or providing information on the workflow that generated the data are some of many examples where metadata annotation (e.g., using $R D F$ ) and externally defined ontologies can lead to increased reproducibility of workflows and reusability of data. JPhyloIO simplifies writing new and extending existing software that is aimed at achieving this goal by fully supporting metadata-rich formats. Maximum interoperability to older software and downwards compatibility is guaranteed by the parallel support for both advanced and more traditional formats, enabled by the single, format-independent interface of JPhyloIO. Developers may support all formats in one step without the need for detailed knowledge on all of them. JPhyloIO's event-based architecture makes integration with any existing application data structure easy and allows very memory-efficient processing even of very large data sets.

\section{Availability and requirements \\ Project name: JPhyloIO.}

Project home page: http://bioinfweb.info/JPhyloIO/

GitHub Repository: https://github.com/bioinfweb/JPhyloIO

ResearchGate project page: http://r.bioinfweb.info/R G)PhyloIO

Operating system(s): Platform independent.

Programming language: Java.

Other requirements: Java Runtime Environment 8 (or higher).

License: GNU Lesser General Public License Version 3 (LGPL).

Any restrictions to use by non-academics: The restrictions specified in the LGPL apply. (See http://bioinf web.info/JPhyloIO/License/LGPL.)

\section{Abbreviations}

API: Application programming interface; CURIE: Compact Uniform Resource Identifier; DFG: Deutsche Forschungsgemeinschaft (German Research Fundation); DNA: Deoxyribonucleic acid; FASTA: Fast Adaptive Shrinkage Thresholding Algorithm (This abbreviation is used here for FASTA alignment format.); GNU: Gnu's Not Unix (Recursive acronym. Uses a wildebeest (Connochaetes) as its icon, which is "Gnu" in German.); GUI: Graphical User Interface; I/O: Input/Output; ID: Identifier; LGPL: Lesser General Public License; NCBI: National Center for Biotechnology Information; OTU: Operational Taxonomic Unit; OWL: Web Ontology Language (The letter reversal is intended by the authors.); PhyDE: Phylogenetic Data Editor; RDF: Resource Description Framework; UML: Unified Modeling Language; XML: Extensible Markup Language; XTG: Extensible TreeGraph format

\section{Acknowledgements}

We are grateful to the anonymous reviewers for their helpful comments. The developers and users of the EDIT platform for Cybertaxonomy at the Berlin Botanical Garden and Botanical Museum tested the integration of JPhylo/O with the Taxonomic Editor, which is highly appreciated. We thank the contributors to the open source projects used by JPhylolO (Apache commons, OWL API, JUnit, Hemcrest).

Parts of this work came from chapter 2 of the PhD thesis of BCS with the title "Software Components for increased Data Reuse and Reproducibility in Phylogenetics and Phylogenomics" that is available at http://nbn-resolving. de/urn:nbn:de:hbz:6-96159516963. Contributions also came from the master thesis of SW with the title "Development and implementation of software components increasing the accessibility of phylogenetic metadata" that is available at http://r.bioinfweb.info/ThesisPhylogeneticMetadata. An earlier version of JPhylo/O was presented in a poster by BCS, SW and KFM with the title "JPhyloIO - A Java library for event-based reading and writing of different alignment and tree formats through one common interface" at the European Conference on Computational Biology (ECCB); The Hague, The Netherlands; 2016 that is available at https://doi.org/10.7490/f1000research.1112 992.1. JPhylolO was also subject of a poster by SW, KFM and BCS with the title "Increasing data accessibility and reuse in phylogenetics by employing externally defined ontologies" that was presented at the 6th annual Symposium of 
the Münster Graduate School of Evolution; Münster, Germany; 2017 and is available at http://r.bioinfweb.info/PosterlncDataAccessReuse.

\section{Authors' contributions}

$\mathrm{BCS}$ conceived the concept, implemented the software and wrote the manuscript; SW contributed to the implementation the software; KFM and SW contributed to the manuscript. All authors gave final approval for publication.

\section{Funding}

Funded in part by grant MU 2875/3-1 to Kai Müller by the German research foundation (DFG). The authors acknowledge support from the Open Access Publication Fund of the University of Münster. The funding bodies did not influence the design of the study and collection, analysis, and interpretation of data or writing the manuscript.

\section{Availability of data and materials}

Binary distributions of JPhylo/O are available at http://bioinfweb.info/ JPhylolO/Download. Source codes are available at http://bioinfweb.info/ JPhylolO/SourceCode or at the GitHub mirror at https://github.com/ bioinfweb/JPhylolO. Unit tests and test data that was used in the development of JPhylo/O is available at http://r.bioinfweb.info/JPhylolOTests.

\section{Ethics approval and consent to participate}

Not applicable.

\section{Consent for publication}

Not applicable.

\section{Competing interests}

The authors declare that they have no competing interests.

Received: 17 May 2019 Accepted: 2 July 2019

Published online: 22 July 2019

\section{References}

1. Kelling S, Hochachka WM, Fink D, Riedewald M, Caruana R, Ballard G, et al. Data-intensive science: a new paradigm for biodiversity studies. BioScience. 2009:59:613-20

2. Michener WK, Jones MB. Ecoinformatics: supporting ecology as a data-intensive science. Trends Ecol Evol. 2012;27:85-93.

3. McKain MR, Johnson MG, Uribe-Convers S, Eaton D, Yang Y. Practical considerations for plant phylogenomics. Appl Plant Sci. 2018:6:e1038.

4. Geiger MF, Astrin JJ, Borsch T, Burkhardt U, Grobe P, Hand R, et al. How to tackle the molecular species inventory for an industrialized nation - lessons from the first phase of the German barcode of life initiative GBOL (20122015). Genome. 2016;59:661-70.

5. Ratnasingham S, Hebert PDN. Bold: the barcode of life data system (http:// www.barcodinglife.org). Mol Ecol Notes 2007;7:355-364.

6. Kerr KC, Stoeckle MY, Dove CJ, Weigt LA, Francis CM, Hebert PD. Comprehensive DNA barcode coverage of north American birds. Mol Ecol Resour. 2007;7:535-43.

7. Global Plants on JSTOR. https://plants.jstor.org/. Accessed 18 Jun 2019.

8. Smith V, Blagoderov V. Bringing collections out of the dark. ZooKeys. 2012;209:1-6.

9. Dickinson JL, Zuckerberg B, Bonter DN. Citizen science as an ecological research tool: challenges and benefits. Annu Rev Ecol Evol Syst. 2010;41: 149-72

10. Hochachka WM, Fink D, Hutchinson RA, Sheldon D, Wong W-K, Kelling S. Data-intensive science applied to broad-scale citizen science. Trends Ecol Evol. 2012;27:130-7.

11. Joly A, Goëau H, Bonnet P, Bakić V, Barbe J, Selmi S, et al. Interactive plant identification based on social image data. Ecol Inform. 2014;23:22-34.

12. Schröter M, Kraemer R, Mantel M, Kabisch N, Hecker S, Richter A, et al. Citizen science for assessing ecosystem services: status, challenges and opportunities. Ecosyst Serv. 2017;28:80-94.

13. Stoltzfus A, O'Meara B, Whitacre J, Mounce R, Gillespie EL, Kumar S, et al. Sharing and re-use of phylogenetic trees (and associated data) to facilitate synthesis. BMC Res Notes. 2012:5:574.

14. Rambaut A. TreeThief: a tool for manual phylogenetic tree entry from scanned images. Department of Zoology, University of Oxford. Oxford. Available from: http://microbe. bio. indiana. edu; 1999
15. Hughes J. TreeRipper web application: towards a fully automated optical tree recognition software. BMC Bioinformatics. 2011;12:178.

16. Laubach T, von Haeseler A, Lercher MJ. TreeSnatcher plus: capturing phylogenetic trees from images. BMC Bioinformatics. 2012:13:110.

17. Murray-Rust $P$, Smith-Unna R, Mounce R. AMI-diagram: mining facts from images. -Lib Mag. 2014;20 11/12. doi:https://doi.org/10.1045/november14murray-rust

18. Cranston $\mathrm{K}$, Harmon LJ, O'Leary MA, Lisle C. Best practices for data sharing in phylogenetic research. PLOS Curr Tree Life. 2014. https://doi.org/10.1371/ currents.tol.bf01eff4a6b60ca4825c69293dc59645 .

19. Poisot TE, Mounce R, Gravel D. Moving toward a sustainable ecological science: don't let data go to waste! Ideas Ecol Evol. 2013;6.

20. Parr CS, Guralnick R, Cellinese N, Page RDM. Evolutionary informatics: unifying knowledge about the diversity of life. Trends Ecol Evol. 2012; 27:94-103.

21. Kenall A, Harold S, Foote C. An open future for ecological and evolutionary data? BMC Evol Biol. 2014;14:66.

22. Piel WH, Chan L, Dominus MJ, Ruan J, Vos RA, Tannen V. TreeBASE v. 2: a database of phylogenetic knowledge. In: e-BioSphere 2009. London; 2009. https://treebase.org/. Accessed 28 Feb 2017

23. National Evolutionary Synthesis Center, UNC-CH Metadata Research Center, Oxford University, The British Library. Dryad. Dryad. https://datadryad.org/. Accessed 18 Jun 2019

24. Stoltzfus A, Lapp H, Matasci N, Deus H, Sidlauskas B, Zmasek CM, et al. Phylotastic! Making tree-of-life knowledge accessible, reusable and convenient. BMC Bioinformatics. 2013;14:158.

25. Hinchliff CE, Smith SA, Allman JF, Burleigh JG, Chaudhary R, Coghill LM, et al. Synthesis of phylogeny and taxonomy into a comprehensive tree of life. Proc Natl Acad Sci. 2015;112:12764-9.

26. Kumar S, Stecher G, Suleski M, Hedges SB. TimeTree: a resource for timelines, Timetrees, and divergence times. Mol Biol Evol. 2017;34:1812-9.

27. White EP, Baldridge E, Brym ZT, Locey KJ, McGlinn DJ, Supp SR. Nine simple ways to make it easier to (re) use your data. Ideas Ecol Evol. 2013;6. https:// doi.org/10.4033/ieev6i2.4608

28. Whitlock MC. Data archiving in ecology and evolution: best practices. Trends Ecol Evol. 2011;26:61-5.

29. Federhen S. The NCBI taxonomy database. Nucleic Acids Res. 2012;40:D136-43.

30. Kilian N, Henning T, Plitzner P, Müller A, Güntsch A, Stöver BC, et al. Sample data processing in an additive and reproducible taxonomic workflow by using character data persistently linked to preserved individual specimens. Database. 2015;2015:bav094.

31. Sandve GK, Nekrutenko A, Taylor J, Hovig E. Ten simple rules for reproducible computational research. PLoS Comput Biol. 2013:9:e1003285.

32. Leebens-Mack J, Vision T, Brenner E, Bowers JE, Cannon S, Clement MJ, et al. Taking the first steps towards a standard for reporting on phylogenies: minimum information about a phylogenetic analysis (MIAPA). OMICS J Integr Biol. 2006:10:231-7.

33. Hang ST, Tatsuma A, Aono M. Bluefield (kde tut) at lifeclef 2016 plant identification task. In: Working Notes of CLEF 2016 conference; 2016. http://ceur-ws.org/Vol-1609/16090459.pdf. Accessed 14 Sep 2016.

34. Wilf P, Zhang S, Chikkerur S, Little SA, Wing SL, Serre T. Computer vision cracks the leaf code. Proc Natl Acad Sci. 2016;113:3305-10.

35. Barré $P$, Stöver BC, Müller KF, Steinhage V. LeafNet: a computer vision system for automatic plant species identification. Ecol Inform. 2017; 40:50-6.

36. Felsenstein J. Phylip input files. 2013. http://evolution.genetics.washington edu/phylip/doc/main.html\#inputfiles. Accessed 18 Jun 2019.

37. Felsenstein J. The Newick tree format. 1993. http://evolution.genetics. washington.edu/phylip/newicktree.html. Accessed 18 Jun 2019.

38. Maddison DR, Swofford D, Maddison WP. NEXUS: an extensible file format for systematic information. Syst Biol. 1997;46:590-621.

39. Vos RA, Balhoff JP, Caravas JA, Holder MT, Lapp H, Maddison WP, et al. NeXML: rich, extensible, and verifiable representation of comparative data and metadata. Syst Biol. 2012:61:675-89.

40. Han MV, Zmasek CM. phyloXML: XML for evolutionary biology and comparative genomics. BMC Bioinformatics. 2009:10:356.

41. RDF - Semantic Web Standards. https://www.w3.org/RDF/. Accessed 18 Jun 2019

42. Wang X, Gorlitsky R, Almeida JS. From XML to RDF: how semantic web technologies will change the design of "omic" standards. Nat Biotechnol. 2005:23:1099-103. 
43. Gamma E, Helm R, Johnson RE, Vlissides J. Design patterns. Elements of reusable object-oriented software. 1st ed. Reprint. Reading, Mass: Prentice Hall; 1994.

44. Miller M, Schwartz T, Pfeiffer W. Relaxed PHYLIP format. Relaxed PHYLIP format documentation at CIPRES. 2016. http://www.phylo.org/index.php/ help/relaxed_phylip. Accessed 18 Jun 2019.

45. Maddison WP, Maddison DR. Mesquite: a modular system for evolutionary analysis. 2016. http://mesquiteproject.org/. Accessed 18 Jun 2019.

46. Ronquist F, Teslenko M, van der Mark P, Larget B, Donald S, Huelsenbeck J. The MrBayes Input Format. 2016. http://mrbayes.sourceforge.net/Help/ format.html. Accessed 18 Jun 2019.

47. Ronquist F, Teslenko M, van der MP, Ayres DL, Darling A, Höhna S, et al. MrBayes 3.2: efficient Bayesian phylogenetic inference and model choice across a large model space. Syst Biol. 2012;61:539-42.

48. Kumar S, Stecher G, Tamura K. MEGA7: molecular evolutionary genetics analysis version 7.0 for bigger datasets. Mol Biol Evol. 2016:msw054.

49. Cardona G, Rosselló F, Valiente G. Extended Newick: it is time for a standard representation of phylogenetic networks. BMC Bioinformatics. 2008;9:532.

50. Müller J, Müller K, Neinhuis C, Quandt D. PhyDE - Phylogenetic Data Editor. 2006. http://phyde.de/. Accessed 18 Jun 2019.

51. Stöver BC, Müller KF. TreeGraph 2: combining and visualizing evidence from different phylogenetic analyses. BMC Bioinformatics. 2010;11:7.

52. Zmasek CM, Eddy SR. ATV: display and manipulation of annotated phylogenetic trees. Bioinformatics. 2001;17:383-4.

53. Zmasek CM. NHX - New Hampshire eXtended, version 2.0. 2014. https:// sites.google.com/site/cmzmasek/home/software/forester/nhx. Accessed 18 Jun 2019.

54. Bouckaert R, Heled J, Kühnert D, Vaughan T, Wu C-H, Xie D, et al. BEAST 2: a software platform for Bayesian evolutionary analysis. PLoS Comput Biol. 2014;10:e1003537.

55. Vogt L. eScience and the need for data standards in the life sciences: in pursuit of objectivity rather than truth. Syst Biodivers. 2013;11:257-70.

56. Zmasek CM. forester: Software libraries for evolutionary biology and comparative genomics research. 2015. https://sites.google.com/site/ cmzmasek/home/software/forester. Accessed 18 Jun 2019.

57. Maddison DR, Schulz K-S, Maddison WP. The tree of life web project. Zootaxa. 2007;1668 Linnaeus Tercentenary: Progress in Invertebrate Taxonomy:19-40.

58. Prlić A, Yates A, Bliven SE, Rose PW, Jacobsen J, Troshin PV, et al. BioJava: an open-source framework for bioinformatics in 2012 Bioinformatics. 2012;28:2693-5.

59. Holland RCG, Down TA, Pocock M, Prlic A, Huen D, James K, et al. BioJava: an open-source framework for bioinformatics. Bioinformatics. 2008;24:2096-7.

60. Vos RA, Huang D, Midford PE. Balhoff J. Sukumaran J. nexml.java - Java API for NeXML. GitHub: Holder MT; 2016. https://github.com/nexml/nexml.java. Accessed 18 Jun 2019

61. Hladish T, Gopalan V, Liang C, Qiu W, Yang P, Stoltzfus A. Bio::NEXUS: a Perl API for the NEXUS format for comparative biological data. BMC Bioinformatics. 2007;8(1).

62. Boettiger C, Chamberlain S, Vos R, Lapp H. RNeXML: a package for reading and writing richly annotated phylogenetic, character and trait data in $\mathrm{r}$. Methods Ecol Evol. 2016;7:352-7.

63. Stajich JE, Block D, Boulez K, Brenner SE, Chervitz SA, Dagdigian C, et al. The Bioperl toolkit: Perl modules for the life sciences. Genome Res. 2002;12:1611-8

64. Lewis PO. NCL: a C++ class library for interpreting data files in NEXUS format. Bioinformatics. 2003;19:2330-1.

65. Sukumaran J, Holder MT. DendroPy: a Python library for phylogenetic computing. Bioinformatics. 2010;26:1569-71.

66. Vos RA, Caravas J, Hartmann K, Jensen MA, Miller C. BIO::Phylo-phyloinformatic analysis using perl. BMC Bioinformatics. 2011;12:63.

67. Py4J - A Bridge between Python and Java. https://www.py4j.org/index.html. Accessed 18 Jun 2019

68. rJava - Low-level R to Java interface. https://www.rforge.net/rJava/. Accessed 18 Jun 2019

69. Stöver BC, Müller KF. LibrAlign: a GUI library for displaying and editing multiple sequence alignments and attached data. 2016. http://bioinfweb. info/LibrAlign/. Accessed 18 Jun 2019.

70. Berendsohn WG. Devising the EDIT platform for Cybertaxonomy. In: Tools for identifying biodiversity: Progress and problems. Proceedings of the international congress, Paris, September 20-22, 2010. EUT Edizioni Università di Trieste; 2010. http://www.openstarts.units.it/dspace/handle/10077/3737. Accessed 18 Jun 2019.

71. Stöver BC, Müller KF. AlignmentComparator: an application to efficiently visualize and annotate differences between alternative multiple sequence alignments. 2014. http://bioinfweb.info/AlignmentComparator/. Accessed 18 Jun 2019

72. Stöver BC, Bohn J, van Groen S, Kösters L, Quandt D, Müller KF. PhyDE 2 an alignment editor for phylogenetic purposes. PhyDE 2 - an alignment editor for phylogenetic purposes. 2019. http://bioinfweb.info/PhyDE2/ Accessed 18 Jun 2019

73. Preston-Werner T. Semantic Versioning 2.0.0. 2013. https://semver.org/. Accessed 18 Jun 2019

\section{Publisher's Note}

Springer Nature remains neutral with regard to jurisdictional claims in published maps and institutional affiliations.
Ready to submit your research? Choose BMC and benefit from:

- fast, convenient online submission

- thorough peer review by experienced researchers in your field

- rapid publication on acceptance

- support for research data, including large and complex data types

- gold Open Access which fosters wider collaboration and increased citations

- maximum visibility for your research: over $100 \mathrm{M}$ website views per year

At $\mathrm{BMC}$, research is always in progress.

Learn more biomedcentral.com/submissions 